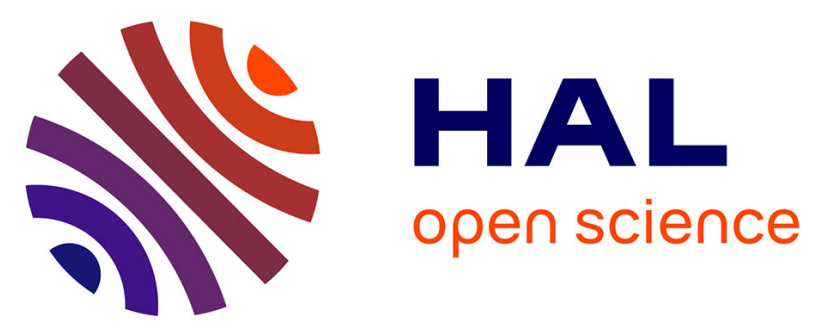

\title{
Smart and Green ACC, adaptation of the ACC strategy for electric vehicle with regenerative capacity
} Sébastien Glaser, Olivier Orfila, Lydie Nouveliere, Roman Potarusov, Sagar Akhegaonkar, Frédéric Holzmann

\section{- To cite this version:}

Sébastien Glaser, Olivier Orfila, Lydie Nouveliere, Roman Potarusov, Sagar Akhegaonkar, et al.. Smart and Green ACC, adaptation of the ACC strategy for electric vehicle with regenerative capacity. IEEE Intelligent Vehicles Symposium (IV 2013), Jun 2013, Gold Coast, Australia. pp.970-975, 10.1109/IVS.2013.6629592 . hal-01009701

\section{HAL Id: hal-01009701 https://hal.science/hal-01009701}

Submitted on 5 Jul 2021

HAL is a multi-disciplinary open access archive for the deposit and dissemination of scientific research documents, whether they are published or not. The documents may come from teaching and research institutions in France or abroad, or from public or private research centers.
L'archive ouverte pluridisciplinaire HAL, est destinée au dépôt et à la diffusion de documents scientifiques de niveau recherche, publiés ou non, émanant des établissements d'enseignement et de recherche français ou étrangers, des laboratoires publics ou privés.

\section{(c)(1)}

Distributed under a Creative Commons Attribution| 4.0 International License 


\title{
Smart and Green ACC, adaptation of the ACC strategy for electric vehicle with regenerative capacity
}

\author{
Sebastien Glaser ${ }^{1}$, Olivier Orfila ${ }^{1}$, Lydie Nouveliere ${ }^{1}$, \\ Roman Potarusov $^{1}$, Sagar Akhegaonkar ${ }^{2}$, Frederic Holzmann ${ }^{2}$, Volker Scheuch ${ }^{2}$
}

\begin{abstract}
This paper presents an optimization of a conventional Adaptive Cruise Control system (ACC) for the specific use of electric vehicles with regenerative capacity, namely the Smart and Green ACC (SAGA). Longitudinal control strategies, that are developed for the driving assistances, mainly aim at optimizing the safety and the comfort of the vehicle occupants. Electric vehicles have the possibility, depending on the architecture, the speed and the braking demand, to regenerate a part of the electric energy during the braking. Moreover, the electric vehicle range is currently limited. The opportunity to adapt the braking of an ACC system to extend slightly the range must not be avoided. When the ACC is active, the vehicle speed is controlled automatically either to maintain a given clearance to a forward vehicle, or to maintain the driver desired speed, whichever is lower. We define how we can optimize both mode and what is the impact, in term of safety and strategy, including the knowledge of the future of the road, integrating a navigation system.
\end{abstract}

\section{INTRODUCTION}

Daily traffic congestion or long trip on a highway are problems that the driver must face during his driving experience. However, these tasks may generate anger and stress. Automation, and automated driving seem to be one possible answer to these problems, by delegating partly or totally the driving task. Many projects during the 80's and 90's, have proved the feasibility of automated driving systems. Eureka Prometheus project in Europe, or the US National Automated Highway System consortium conducted experiments on real road of automated driving or platoon. Even if the concepts were not fully transmitted to car manufacturers, current vehicles benefit greatly from these research: since 10 years, the driving assistances are booming.

The driving assistances help the driver to manage the driving task at different levels. Tom M. Gassner [1] defined several automation level specifically applied to the driving task, which are:

- Driver only: the driver controls the full driving task

- Assisted: the driver controls one dimension and monitors the other one. He must be prepared to take over the complete control at any time.

\footnotetext{
* Research supported by the European Community Seventh Framework program as part of the eFuture project (GA 258133)

${ }^{1}$ Sebastien Glaser, Olivier Orfila, Lydie Nouveliere, Roman Potarusov are with the LIVIC (Laboratory on Interactions Vehicles-InfrastructureDrivers), a research unit of IFSTTAR, Batiment 824, 14, Route de la Miniere, 78000 Versailles, France (phone: +33 1404329 08; e-mail: firstname.lastname@ifsttar.fr).

${ }^{2}$ Sagar Akhegaonkar, Frederic Holzmann are with INTEDIS GmbH \& Co. KG, Max-Mengeringhausen-Strae 5, 97084 Wuerzburg - Germany (phone: +49 (0) 931 6602-35740; e-mail: firstname.lastname@intedis.com).
}

- Partially automated: the system controls the driving task, but the driver monitors the system and may also take over the complete control at any time

- Highly automated: the driver no longer need to permanently monitor the system, but he may still take over the control with a certain time buffer.

- Fully automated: the system is able to fully monitor itself and in case of a takeover request that is not realized within the time buffer, the system may return to the minimum risk situation by itself.

In today situation, driving assistances already address the assisted level, as the Adaptive Cruise Control, which is one of the most relevant. They do not aim at higher level of automation, mainly because of reliability and legal aspect.

With an Adaptive Cruise Control system, the driver delegates the longitudinal control task. When the system is active, the vehicle speed is controlled automatically either to maintain a given clearance to a forward vehicle, or to maintain the driver desired speed, whichever is lower. Since 1997, car manufacturers propose this system on their highend cars. However, the research is still active. Researchers aim at evaluating the impact of the ACC on traffic, under congested situation [2] or with improved strategies [3]. They also extend the range of possible speeds, driver comfort, safety or road capacity [4], [5]. In 2006, the introduction of a vehicle to vehicle communication (cooperation ACC, C-ACC) allows to decrease drastically the clearance to a forward vehicle [6] and also to create stable vehicle platoon. The evaluation of the C-ACC [7], [8] shows promising results on road capacity and safety. An ISO norm now defines the intended performance of the ACC [9].

In parallel, navigation devices became popular. They allow to have access to longer range information on the road and its status, especially with systems that include communication with a traffic management center. Two main families of Advanced Driving Assistance Systems (ADAS) appear. The first one provides warning to the driver, or limit the speed of the vehicle, according with the legal context. This is the Intelligent Speed Adaptation (ISA) system. They prove to be efficient [12], and acceptable. Large scale studies have been done or are currently conduct around the world [13], [14]. the second one uses the map and the geometric description of the road to define a safe speed. These systems are named Curve Warning System, CWS. The driver is warned if his speed is higher than the profile defined previously, he can even be limited or the vehicle can be controlled [15], [16]. These systems now include communication with a traffic 


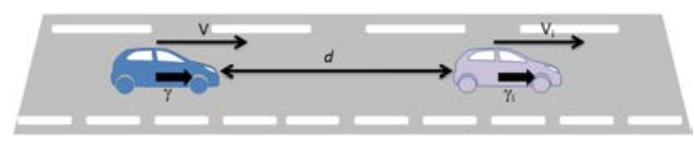

Fig. 1. General use case of the ACC

management center to define legal or safe limit according with dynamic event [17].

At the same time, the energy consumption became one of the major topic in car industry and for the car users: the oil price has skyrocketed and the greenhouse gas emissions must be reduced. The electric vehicles can provide an efficient solution, especially for the commuting. However, they are still limited by their operating range, which can greatly vary with the driver behavior or traffic conditions. The solution that we choose, is to use the ACC in order to have the longitudinal control of the vehicle and to optimize it with energy efficiency consideration. The system will manage short range interaction, with other vehicles, and long range information, on road and possible event. In [20], a driving assistance is developed using a dynamic programming (DP) approach. However, the DP is quite slow and requires a huge computation. We present here a direct computation method, meaning that we compute directly the speed from a differential equation rather than exploring possible state.

In the following, we develop this solution. Section II presents the notation and the ACC, its performance and its limitation. Section III develops the consumption model that is used to evaluate the solution and the motor characteristic. Section IV shows the optimization process that is define and presents the first results. Finally, section V concludes this article.

\section{SAGA DEFINITION}

In this section, we will firstly describe the notations that are used in the following, next the behavior and requirement of an ACC system, and the extension to the Low Speed Following and Full Speed Range ACC, and finally we present the architecture of our system.

\section{A. Notation}

With respect to the longitudinal control, the driver is either on a free driving situation, without any forward vehicle, or constrained by a forward vehicle. This case is shown in the figure 1 . The variables describing the state of each vehicles are defined in the table I. Moreover, the driver may also select the desired speed, $V_{\text {driver }}$ and the desired time headway, $T_{\text {driver }}$

\section{B. ACC behavior and performance}

The common ACC systems [9] use two low level functions:

- Speed Regulation: the system must control its speed around a driver-desired speed. This function is enable when no vehicles are detected in front of our vehicle

- Vehicle Following: the vehicle controls its speed according to the speed of the lead vehicle.
TABLE I

VEHICLE DYNAMIC RELATED VARIABLES FOR THE ACC FUNCTION

\begin{tabular}{llc}
\hline Variable & Name & Unit \\
\hline$d$ & Distance to the forward vehicle & $\mathrm{m}$ \\
$V$ & Speed of the ego vehicle & $\mathrm{m} / \mathrm{s}$ \\
$\gamma$ & Acceleration of the ego vehicle & $\mathrm{m} / \mathrm{s}^{2}$ \\
$T$ & Time Headway $(T=d / V)$ & $\mathrm{s}$ \\
$V_{i}$ & Speed of the forward vehicle & $\mathrm{m} / \mathrm{s}$ \\
$\gamma_{i}$ & Acceleration of the forward vehicle & $\mathrm{m} / \mathrm{s}^{2}$ \\
$\Delta V$ & Relative speed $\left(\Delta V=V_{i}-V\right)$ & $\mathrm{m} / \mathrm{s}$
\end{tabular}

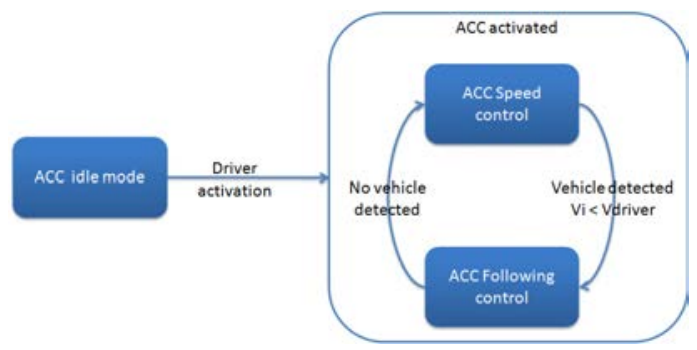

Fig. 2. Strategy of ACC, the ACC switch to an activated mode with a driver request

The switch between each mode is decided using the following criteria (figure 2): The detection, or not of a forward vehicle, the relative speed and the clearance, and finally the driver desired speed. Moreover, [9] also defines the operating range of the driving assistance. The assistance can not be activated below a given speed $V_{\min }$, which must be higher than $7 \mathrm{~m} / \mathrm{s}$. The average automatic deceleration of ACC systems shall not exceed $3.5 \mathrm{~m} / \mathrm{s}^{2}$, while the acceleration is limited to $2 \mathrm{~m} / \mathrm{s}^{2}$. the average rate of change of an automatic deceleration (jerk) shall not exceed $2.5 \mathrm{~m} / \mathrm{s}^{3}$. The ISO norm also defines the minimal performance of the perception system according with the possible value of the speed and of the time gap.

The iso norms [10] and [11] complete the description of the longitudinal control respectively for the low speed following and the full speed range adaptive cruise control (Fig. 3).

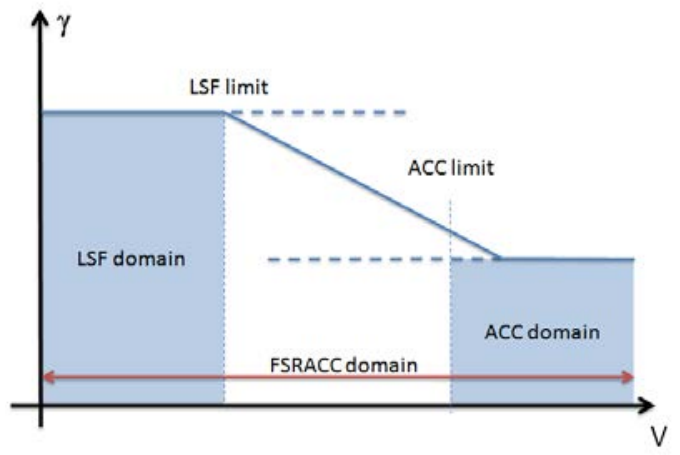

Fig. 3. Operating area of an longitudinal ADAS : Low Speed Following (LSF), Adaptive Cruise Control (ACC) and Full Speed Range ACC (FSRACC) 
TABLE II

MECHANIC AND ELECTRIC ENERGY THAT IS CONSUMED OR REGENERATED, DEPENDING ON THE SITUATION

\begin{tabular}{ccc}
\hline Energy & Consumption & Regeneration \\
\hline Mechanic & $E_{m}=\frac{1}{\eta_{g}} T_{e} w_{e} d t$ & $E_{m}=\eta_{g} T_{e} w_{e} d t$ \\
Electric & $E_{\text {elec }}=\frac{1}{\eta_{b}} E_{m} \eta\left(T_{e}, w_{e}\right)$ & $E_{\text {elec }}=\eta_{b} E_{m} \eta\left(T_{e}, w_{e}\right)$ \\
\hline
\end{tabular}

\section{SAGA architecture}

The aim of the Smart and Green ACC is to provide a longitudinal driving assistance that is safe, comfortable and also efficient. We have to make the distinction between two main subfunctions of the system: the speed regulation and the vehicle following. For this article, we will focus on the problem of speed regulation. The vehicle following task will be discussed in the conclusion.

The speed regulation task needs to define a speed profile that is safe, according with the environment, and efficient. In order to make the system as comfortable as possible, we must define the speed enough in advance. So, the system must know the future of the road, using the vehicle localization (from a GPS) and the geometric description of the road and its limitation (from a digital map). In the following, we will describe the process to achieve a safe and efficient speed profile, and the requirements.

\section{ELECTRIC VEHICLE CONSUMPTION MODEL}

\section{A. Consumption and regeneration model}

In order to accelerate, decelerate, or even keep a constant speed, the vehicle motor, or braking system, must generate a torque on the driven axle. This torque must overcome all resisting forces and generate the acceleration. It could be defined as:

$$
T_{e}=R_{w}\left(\frac{1}{2} \rho S C_{x} V^{2}+M g C_{r r}+M g \sin \phi_{r}+M \gamma\right)
$$

where $R_{w}$ is the wheel radius, $\rho$ the air volumetric mass, $S C_{x}$ the air drag coefficient, $V$ the current vehicle speed, $M$ the vehicle's mass, $C_{r r}$ the rolling resistance coefficient, $\phi_{r}$ the slope and $\gamma$ the vehicle acceleration. The engine speed, supposing without sliding, is $w_{e}=V / R_{w}$. Finally, we evaluate the kinetic energy and the electric energy that is either consumed or regenerated during a time $d t$, depending on the value of the torque, in the table II.

Where $\eta_{g}$ is the transmission efficiency, $\eta_{b}$ is the battery's efficiency and $\eta\left(T_{e}, w_{e}\right)$ the motor's efficiency. This last parameter strongly depends on the motor type. For the evaluation, we will use this simple model to compare the impact of the SAGA system.

\section{B. Regenerative braking area and acceleration limit}

We must also describe the deceleration area where a regenerative braking is possible, depending on the vehicle speed. As a very first approximation, an electric motor is defined by its specific power $P_{e}=T_{e} w_{e}\left(T_{e}\right.$ is defined in 1 , we consider the slope, the rolling resistance, the aerodynamic

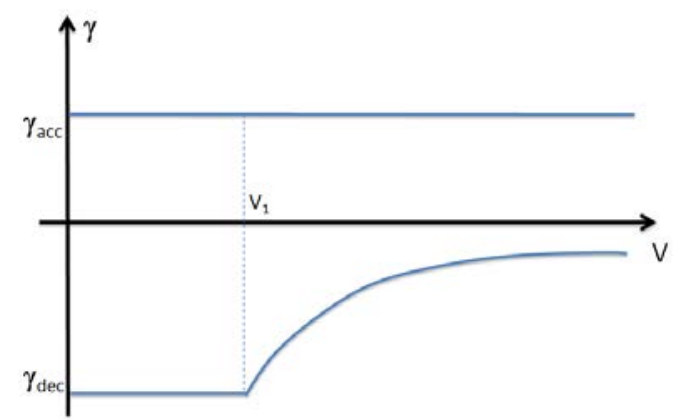

Fig. 4. Possible acceleration domain, considering also regenerative capacity

forces and the desired acceleration). We can link the torque to the acceleration and the engine speed to the vehicle speed. The regenerative deceleration domain could be approximated by a maximal deceleration $\gamma_{d e c}$ below a given value of the speed $V_{1}$. For speeds that are higher, the deceleration is limited by the power of the motor:

$$
\gamma_{d}(V)=\left\{\begin{array}{lll}
\gamma_{d e c} & : & V<V_{1} \\
\frac{A}{V} & : & V \geq V_{1}
\end{array}\right.
$$

where $A$ is a negative constant that depends on the motor and drive train characteristics.

At the opposite, we also limit the possible acceleration to a maximal value $\gamma_{a c c}$. Figure 4 summarizes the possible acceleration domain, limited by the regenerative capacity.

In the Figure 5, we present a real characteristic of regenerative deceleration that is obtained on the first version of the prototype of the EU project eFuture [18]. The simple model that is developed previously, matches the shape of the real curve for speeds that are higher than $8 \mathrm{~m} / \mathrm{s}$. Below $8 \mathrm{~m} / \mathrm{s}$, the regenerative deceleration drops quickly. The main reasons are technological choices and to avoid that the engine generates a torque that is too strong on the wheel and blocks them. This could generate dynamic instability or increase the braking distance.

Moreover, at low speed, the electric energy that could be regenerated is lower than at higher speed because: the mechanic energy is small because the engine speed and possible torque are lower. At lower speed, it is more effective to brake using the conventional braking system: the benefit in term of regeneration is small and we can have stronger braking forces.

\section{OPTIMIZATION PROCESS AND INTEGRATION CONSTRAINTS}

\section{A. Rules for defining a safe speed profile}

The driving rules availability relies on the data included in the digital map. The minimal set of attributes does correspond with the legal speed. In order to define the maximal speed profile, we include the following rules:

- Safety, the vehicle speed profile must comply with these rules:

- Speed is limited by the legal speed limit, 


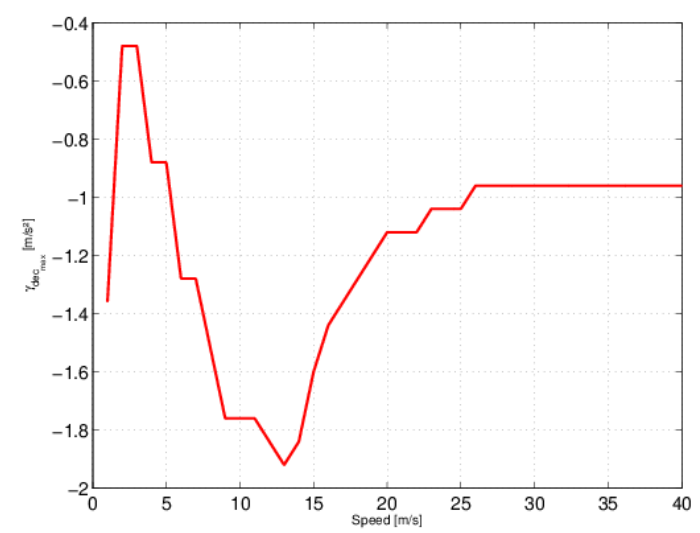

Fig. 5. Real regenerative deceleration capacity of an electric vehicle

- At the position of a stop sign, the vehicle speed must be zero

- Comfort, the following rules may be extended. A test must be done to evaluate the driver acceptance:

- When crossing an intersection, on a main road, the maximal speed is decreased by $10 \mathrm{~km} / \mathrm{h}$

- When crossing an intersection, on a secondary road, the maximal speed is decreased by $20 \mathrm{~km} / \mathrm{h}$

- When approaching an intersection with a traffic light, the maximal speed is decreased by $10 \mathrm{~km} / \mathrm{h}$

\section{B. description of the optimization process}

When the driver, or an automate, controls the vehicle, he mobilizes a given road friction at the level of the tire road interface:

$$
\mu_{m o b}=\frac{F_{x y}}{F_{z}}=\sqrt{\frac{F_{x}^{2}+F_{y}^{2}}{F_{z}^{2}}}
$$

where $F_{x}, F_{y}$ and $F_{z}$ are respectively the forces at the tire road interface in longitudinal, lateral and vertical direction, which can be either on the front (labeled $f$ ) or rear $(r)$ axle. The tire road forces can be defined using the desired acceleration (longitudinal $\gamma_{l o n}$, lateral $\gamma_{l a t}$ and vertical $\gamma_{v e r t}$ ) and the road attributes (slope $\phi_{r}$, banking $\theta_{r}$, and curvature $\left.\rho_{r}\right)$ :

$$
\left\{\begin{array}{l}
M \gamma_{l a t}=F_{y f}+F_{y r}-M g \sin \theta_{r} \\
I \ddot{\psi}=L_{f} F_{y f}-L_{r} F_{y r} \\
\\
M \gamma_{v e r t}=F_{z f}+F_{z r}+M g \cos \phi_{r} \\
I_{y} \ddot{\theta}=H F_{e}-L_{f} F_{z f}+L_{r} F_{z r} \\
M \gamma_{l o n}=F_{e}+M g \sin \phi_{r}
\end{array}\right.
$$

$M$ is the vehicle mass and $F_{e}$ is the force that is generated by the engine to achieve a given acceleration, $I_{y}$ and $I_{z}$ are the inertia of the vehicle body along the $y$ and $z$ axle respectively, $L_{r}, L_{f}$ and $H$ are the distance of the center of gravity to the rear axle, front axle and the height, respectively. Moreover, we can express the lateral acceleration to follow the trajectory defined by the road and the longitudinal acceleration as the desired speed variation:

$$
\left\{\begin{array}{l}
\gamma_{l a t}=\rho_{r} V^{2} \\
M \gamma_{l o n}=V \frac{d V}{d s}
\end{array}\right.
$$

By limiting the longitudinal and lateral maximal mobilized friction to $\lambda_{\text {lon }} \mu_{\max }$ and $\lambda_{\text {lat }} \mu_{\max }$, the expression of the speed is given by the following system ${ }^{1}$ :

$$
\left\{\begin{array}{l}
V^{2}=\frac{g}{\rho_{r}}\left(\left(1+\frac{H}{L_{r}} \phi_{r}\right) \cdots\right. \\
\left.\cdots \sqrt{1-\left(\frac{\phi_{r}}{\lambda_{\text {lon }} \mu_{\text {max }}}\right)^{2}} \lambda_{\text {lat }} \mu_{\text {max }}-\theta_{r}\right) \\
1=\left(\frac{1}{\lambda_{\text {lat }} \mu_{\max }} \frac{\frac{\rho_{r} V^{2}}{g}+\theta_{r}}{1-\frac{H}{L_{r}}\left(\frac{V}{g} \frac{d V}{d s}-\phi_{r}\right)}\right)^{2}+\ldots \\
\ldots\left(\frac{\frac{V}{g} \frac{d V}{d s}-\phi_{r}}{\lambda_{\text {lon }} \mu_{\max }}\right)^{2}
\end{array}\right.
$$

As we limit the deceleration domain, it has an impact on both maximal safe speed and on the differential equation to compute the speed profile: the parameter $\lambda_{l o n} \mu_{\max }$ evolves with the speed for the deceleration phase (see eq. 2):

$$
\lambda_{\text {lon }} \mu_{\max }=\frac{\gamma_{d}(V)}{g}
$$

\section{Optimization and integration constraints}

In the frame of the EU project eFuture, the proposed ADAS must be integrated in the vehicle on an automotive ECU. The ECU is limited both in term of computational power and also in term of memory size. These two constraints limit us for a real optimization process.

The CWS, described previously [15], aims to optimize the use of the road friction, both on acceleration / deceleration phase and during the curve following. It integrates a GPS to localize the system and a map to define the electronic horizon. This electronic horizon does contain the geometric description of the road, including the curvature, the slope and the superelevation. By integrating the deceleration limit to the regenerative deceleration (see figure 5), we can say that the speed profile is the fastest possible according to the safe criteria, that allows the battery regeneration.

However, in [19], we have compared the method to generate the speed profile with existing optimization process, using Dijkstra and $A *$ theories. The cost function $C F$ for the optimization process is:

$$
C F=C_{1} E_{\text {elec }}^{\text {norm }}+C_{2} t^{\text {norm }}
$$

Where $E_{\text {elec }}^{\text {norm }}$ and $t^{\text {norm }}$ are the normalized values of $E_{\text {elec }}$ and $t$, respectively. $t$ is the time needed to reach the speed profile starting from the current vehicle speed $V . C_{1}$ and $C_{2}$ are the weight coefficients. The method that we present here is very close to front of the Pareto space representation of the optimization using the Dijkstra or $A *$ algorithms.

\footnotetext{
${ }^{1}$ More details on the hypothesis and developments could be found in [15]
} 

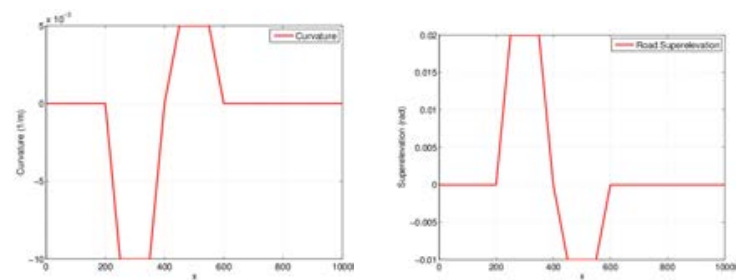

Fig. 6. Road geometry (left: curvature, right: superelevation)

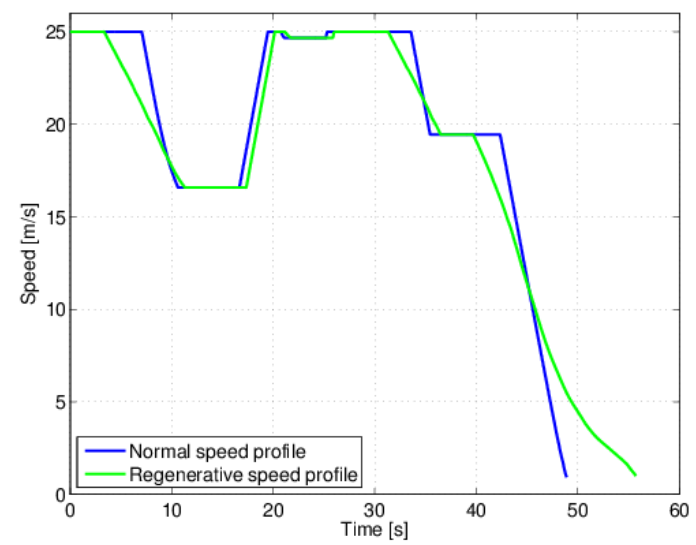

Fig. 7. Speed profiles that result from [15] and the proposed method

\section{Simulation results}

In order to demonstrate this approach, we have defined the following road profile presented in the Figure 6 . The road superelevation is correctly define, given the sign of the curvature. The legal speed limit is $90 \mathrm{~km} / \mathrm{h}$ from 0 to $700 \mathrm{~m}$, and then drops at $70 \mathrm{~km} / \mathrm{h}$. At $1000 \mathrm{~m}$, there is a stop, the legal speed is $0 \mathrm{~km} / \mathrm{h}$.

We compare the speed that is generated by [15] and the speed from SAGA. In the Figures 7 and 8, the two versions of the generation of the speed profile and the state of charge are presented. We can see that the $1 \mathrm{~km}$ trip needs $48,9 \mathrm{~s}$ for [15] and $55.7 s$ for the SAGA, a $7 s$ difference. The deceleration phase differs between the two profiles, but we choose to keep the acceleration at the same level. Then, the two curves are parallel in the acceleration phase (for instance, before

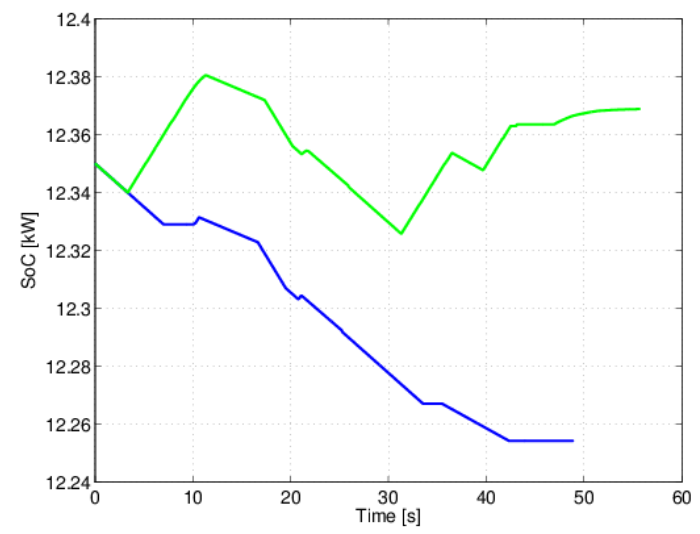

Fig. 8. Resulting battery State of Charge variations

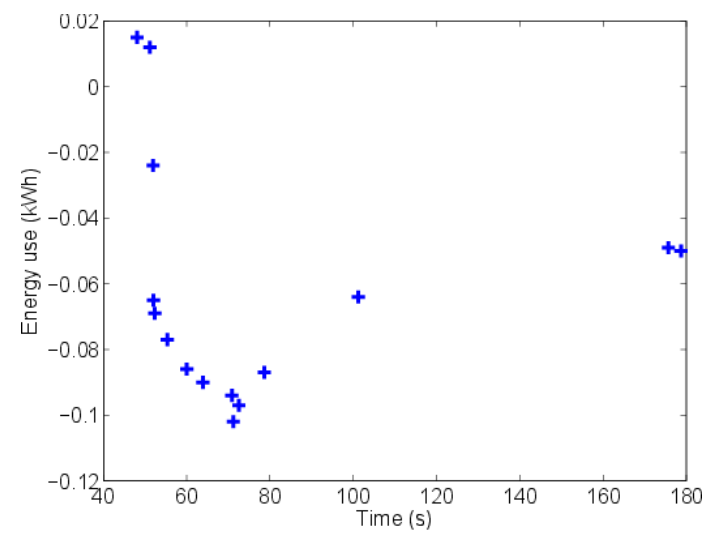

Fig. 9. Result's graph for the $A^{*}$ optimization process and Pareto front

$t=20 \mathrm{~s}$ ). This choice leads to a same variation of the state of charge (see before $t=20 \mathrm{~s}$ ). For the State of Charge, the first speed profile will consume around $0.1 \mathrm{~kW}$, while the SAGA regenerate $0.02 \mathrm{~kW}$. At the end of the curve, the energy that is generated is very low: the motor efficiency is small and the mechanic energy that needs to be absorbed is also small. Moreover, the braking force is not high, according with the Figure 5. Then, we choose to enhance the system by stopping the regenerative deceleration below a given speed threshold and switching to a conventional braking that allows a higher deceleration. For the following, we choose a switching speed of $V_{s}=10 \mathrm{~m} / \mathrm{s}^{2}$. Using this threshold, we gain about $5 \mathrm{~s}$ on the trip duration $(50.8 s)$. At the same time, the energy that is regenerated, remains equivalent.

Figure 9 shows the result's graph for the $A *$ algorithm using the cost function described in eq. 8 on the same road profile. Each point does correspond with a selection of costs in the eq. 8. A Pareto front is clearly visible as an hyperbolic function. Our process generates a speed profile that achieves the trip in $50.8 \mathrm{~s}$, with an energy consumption of $-0.02 \mathrm{~kW}$. We are close to the Pareto front in the graph.

\section{CONCLUSION}

\section{A. SAGA as an energy efficient driving assistance}

We have presented, in this article, an efficient way to supervise longitudinal driving assistance, such as an ACC or FSRACC, with the consideration of legal speed limit, safety and consumption. The proposed method defines a speed profile that can override the maximal speed defined by the driver. The computation of the speed profile takes into account the road geometry provided by a map, the possible regenerative deceleration during braking from the motor definition and a set of traffic rules. Following this speed profile, the trip duration is slightly increased but it allows a regeneration. In term of efficiency, the resulting profile is close to the best results of an $A *$ algorithm. In term of computation time, the speed profile is directly computed and does not need an exploration as for the optimization method: it does not require a lot of computation or memory space. However, the system can not be tuned as an optimization method where the user can select the desired weight for the 
cost function. The next step will be to integrate this version of a Smart and Green ACC (SAGA) on an embedded ECU in a car.

\section{B. Extension to the vehicle following case}

In this article, we limit the SAGA to the speed regulation case, and we keep the vehicle following case as it is described in the ISO norm. This solution allows to have a safe behavior of the driving assistance regarding the other vehicle. In order to allow a regenerative braking, while being in a vehicle following mode, the driving assistance must either take a larger inter vehicular distance or rely on a secondary driving assistance that can generate a braking force that is stronger than conventional ACC, in order to ensure the vehicle safety and no collision on the operating range.

\section{REFERENCES}

[1] Tom M. Gasser, Daniel Westhoof, BASt-study: definition of automation and legel issues in Germany, TRB Road Vehicle automation workshop, 25 july 2012

[2] Greg Marsden, Mike McDonald, Mark Brackstone, Greg Marsden, Towards an understanding of adaptive cruise control, Transportation Research Part C 9 (2001) 33-51

[3] Arne Kesting, Martin Trieber, Martin Schonhof, Dirk Helbing, Extending adaptive cruise control to adaptive driving strategies, Transportation Research Record: Journal of the transportation research board, N2000, 2007, pp 16-24

[4] Ardalan Vahidi, Azim Eskandarian, Research advances in intelligent collision avoidance and adaptive cruise control, IEEE Transactions on Intelligent Transportation Systems, 4(3), 143153.

[5] Seungwuk Moon, Ilki Moon, Kyongsu Yi, Design, tuning,and evaluation of a full-range adaptive cruise control system with collision avoidance, Control Engineering Practice (2009)442455

[6] Gerrit Naus, Ren Vugts, Jeroen Ploeg, Ren van de Molengraft, Maarten Steinbuch, Cooperative Adaptive Cruise Control, IEEE Automotive Engineering Symposium Eindhoven, The Netherlands, April 6, 2009

[7] B. Van Arem, C.J.G. Van Driel and R. Visser, The impact of cooperative adaptive cruise control on traffic flow characteristics, IEEE Transactions on Intelligent Transportation Systems, Vol. 7, No. 4, pp. 429436, Dec. 2006

[8] Christopher Nowakowski, Steven E. Shladover, Delphine Cody et al., Cooperative Adaptive Cruise Control: Testing Drivers Choices of Following Distances, UCB-ITS-PRR-2010-39

[9] ISO 15622:2010, Intelligent transport system, Adaptive Cruise Conrol system, performance requirements and test procedures.

[10] ISO 22178:2009, Intelligent transport system, low speed following systems, performance requirements and test procedures.

[11] ISO 22179:2009, Intelligent transport system, Full speed range adaptive cruise control system, performance requirements and test procedures.

[12] O.M.J Carsten, F.N. Tate, Intelligent Speed Adaptation: Accident Savings and Cost-Benefit Analysis, Accident Analysis and Prevention 37, pp. 4074162005.

[13] LAVIA French project, http://www.lavia.fr/

[14] EuroFOT EU project, http://www.eurofot-ip.eu/

[15] S. Glaser, V. Aguilera, Vehicle Infrastructure Driver Speed Profile : Towards the next Generation of Curve Warning Systems, In Proc. of the 10th ITS World Congress, Madrid, Spain, nov. 2003.

[16] V. Aguilera, S. Glaser, A. Von Arnim, An advanced Driver Speed Assistance in Curves : risk function, cooperation modes, system architecture and experimental Validation. In Proc. of the IEEE Intelligent Vehicle Symposium, Las Vegas, 2005.

[17] SAFESPOT EU Project, http://www.safespot-eu.org/

[18] eFuture EU project, http://www.efuture-eu.org

[19] Sebastien Glaser, Olivier Orfila, Roman Potarusov, Lydie Nouveliere, Speed profile optimization for electric vehicle with regenerative capacity, proposed at FAST-Zero'13, Nagoya, Japan.

[20] Sagar Akhegaonkar, Lydie Nouvelire, Roman Potarusov, Volker Scheuch, Frdric Holzmann, Sbastien Glaser, Modeling and Simulation of Battery Electric Vehicle to Develop an Energy Optimization Algorithm, AVEC 2012, Seoul, Korea 